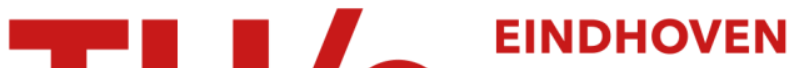

\section{Application of cell mapping methods to a discontinuous dynamic system}

\section{Citation for published version (APA):}

Spek, van der, J. A. W., de Hoon, C. A. L., Kraker, de, A., \& Campen, van, D. H. (1994). Application of cell mapping methods to a discontinuous dynamic system. Nonlinear Dynamics, 6(1), 87-99.

https://doi.org/10.1007/BF00045434

DOI:

10.1007/BF00045434

Document status and date:

Published: 01/01/1994

\section{Document Version:}

Publisher's PDF, also known as Version of Record (includes final page, issue and volume numbers)

\section{Please check the document version of this publication:}

- A submitted manuscript is the version of the article upon submission and before peer-review. There can be important differences between the submitted version and the official published version of record. People interested in the research are advised to contact the author for the final version of the publication, or visit the $\mathrm{DOI}$ to the publisher's website.

- The final author version and the galley proof are versions of the publication after peer review.

- The final published version features the final layout of the paper including the volume, issue and page numbers.

Link to publication

\section{General rights}

Copyright and moral rights for the publications made accessible in the public portal are retained by the authors and/or other copyright owners and it is a condition of accessing publications that users recognise and abide by the legal requirements associated with these rights.

- Users may download and print one copy of any publication from the public portal for the purpose of private study or research.

- You may not further distribute the material or use it for any profit-making activity or commercial gain

- You may freely distribute the URL identifying the publication in the public portal.

If the publication is distributed under the terms of Article 25fa of the Dutch Copyright Act, indicated by the "Taverne" license above, please follow below link for the End User Agreement:

www.tue.nl/taverne

Take down policy

If you believe that this document breaches copyright please contact us at:

openaccess@tue.nl

providing details and we will investigate your claim. 


\title{
Application of Cell Mapping Methods to a Discontinuous Dynamic System
}

\author{
J. A. W. VAN DER SPEK, C. A. L. DE HOON, A. DE KRAKER, and \\ D. H. VAN CAMPEN \\ Department of Mechanical Engineering, Eindhoven University of Technology, P. O. Box 513, $5600 \mathrm{MB}$ \\ Eindhoven, The Netherlands
}

(Received: 10 November 1992; accepted: 26 January 1993)

\begin{abstract}
The Cell Mapping method is a robust tool for investigating nonlinear dynamic systems. It is capable of finding the attractors and corresponding basins of attraction of a system under investigation. To investigate the applicability of the Cell Mapping method to discontinuous systems, a "forced zero-stiffness impact oscillator" is chosen as an application. The numerical integration algorithm, the basic element in the Cell Mapping method, is adjusted to overcome the discontinuity. Four types of Cell Mapping techniques are applied: Simple Cell Mapping, Generalized Cell Mapping, Interpolated Cell Mapping, and Mixed Cell Mapping. The last type is a new modification to existing types. Each type of Cell Mapping is briefly explained. The results are compared to the exact solutions. The Interpolated Cell Mapping and Mixed Cell Mapping methods are found to produce the most accurate results for this case.
\end{abstract}

Key words: Discontinuous systems, chaos, basins of attraction, cell mapping.

\section{Introduction}

The long term behavior of a dissipative nonlinear dynamic system loaded by a periodic force depends on the system's initial state. This is essential for nonlinear systems. When both periodic and chaotic motions are possible for a certain set of system parameters, the initial condition of the system even determines whether the system will behave in a regular (periodic) or chaotic way. Therefore, when investigating a nonlinear system, knowledge of the attractors is not enough. Additional research on the attractors' basins of attraction is required to obtain a complete picture of a system's dynamic behavior.

The attractors and corresponding basins of attraction of a nonlinear system can be found with the method of Cell Mapping, which was established by Hsu [2,3]. This method is based on a discretization of the system's state space in so-called cells. We distinguish between the Simple Cell Mapping (SCM) method and the Generalized Cell Mapping (GCM) method (Hsu [3]). Later, Tongue presented an improved method, the so-called Interpolated Cell Mapping (ICM) method. In [6, 7], this method is introduced and compared to the SCM and GCM methods. In Section 6, a new modification is introduced, termed Mixed Cell Mapping (MCM).

When applying Cell Mapping methods to discontinuous dynamic systems, some modifications need to be made. This is shown on the basis of a "forced zero-stiffness impact oscillator", a dynamic system with a discontinuity. This system is discussed in the next section and treated 

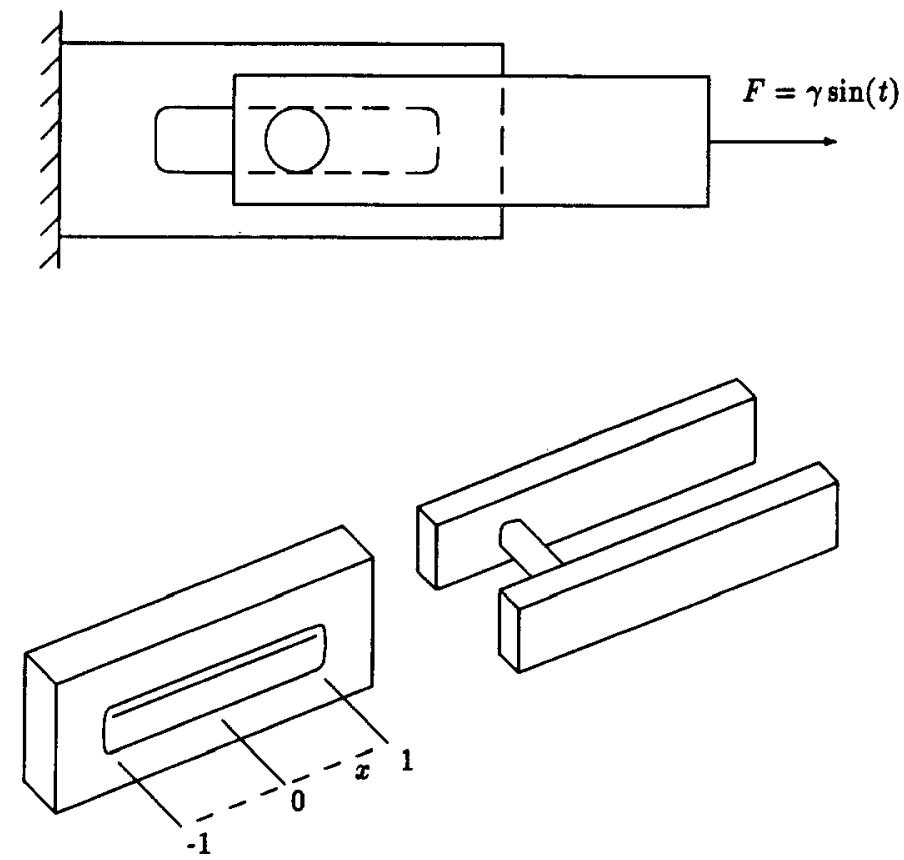

Fig. 1. Mechanical oscillator consisting of two parts, joined by a smooth pin with play, studied by Li et al. [5].

by the Cell Mapping methods in the following sections. The obtained results are found to match with the exact solutions quite well.

\section{A Forced Zero-Stiffness Impact Oscillator}

The behavior of a simple mechanical oscillator is considered. The system consists of two parts: one fixed, having a slot, and one constrained to translate along a straight line, joined by a smooth pin with play (Figure 1). The movable part is excited by a periodic force and encounters no resistance except inertia until the pin reaches the end of the slot. The impact of the pin on the fixed member is inelastic and is modelled by a coefficient of restitution $r$, with $0<r<1$. The system is governed by the following nondimensional differential equation and boundary conditions:

$$
\begin{array}{ll}
x^{\prime \prime}=\gamma \sin (t) & \text { for } \\
x^{\prime} \rightarrow-r x^{\prime} & \text { for }|x|<1,
\end{array}
$$

Here, $x$ measures the position of the pin and $\gamma$ is the forcing amplitude.

This system was studied by Li et al. [5], who showed the existence of periodic solutions for certain values of $r$ and $\gamma$ by means of analytical techniques. For instance, for $r=0.5$ and $\gamma=0.20826$ a periodic motion was found with period $2 \pi \sec$ (Figure 2).

However, we found that chaotic behavior is also possible for the same parameter values. Integration of (1) with initial conditions $x=x^{\prime}=0$ yields a chaotic trajectory, shown in Figure 3a for $t=0-500 \mathrm{sec}$. In Figure 3b, a Poincaré section is shown of both the periodic and chaotic motions. The coordinates of the chaotic trajectory have been plotted at $t=2 n \pi$ sec, $n=100,101, \ldots, 5000$.

In the following sections, we apply the cell mapping methods to the impact oscillator in order to determine all existing solutions as well as their basins of attraction. 


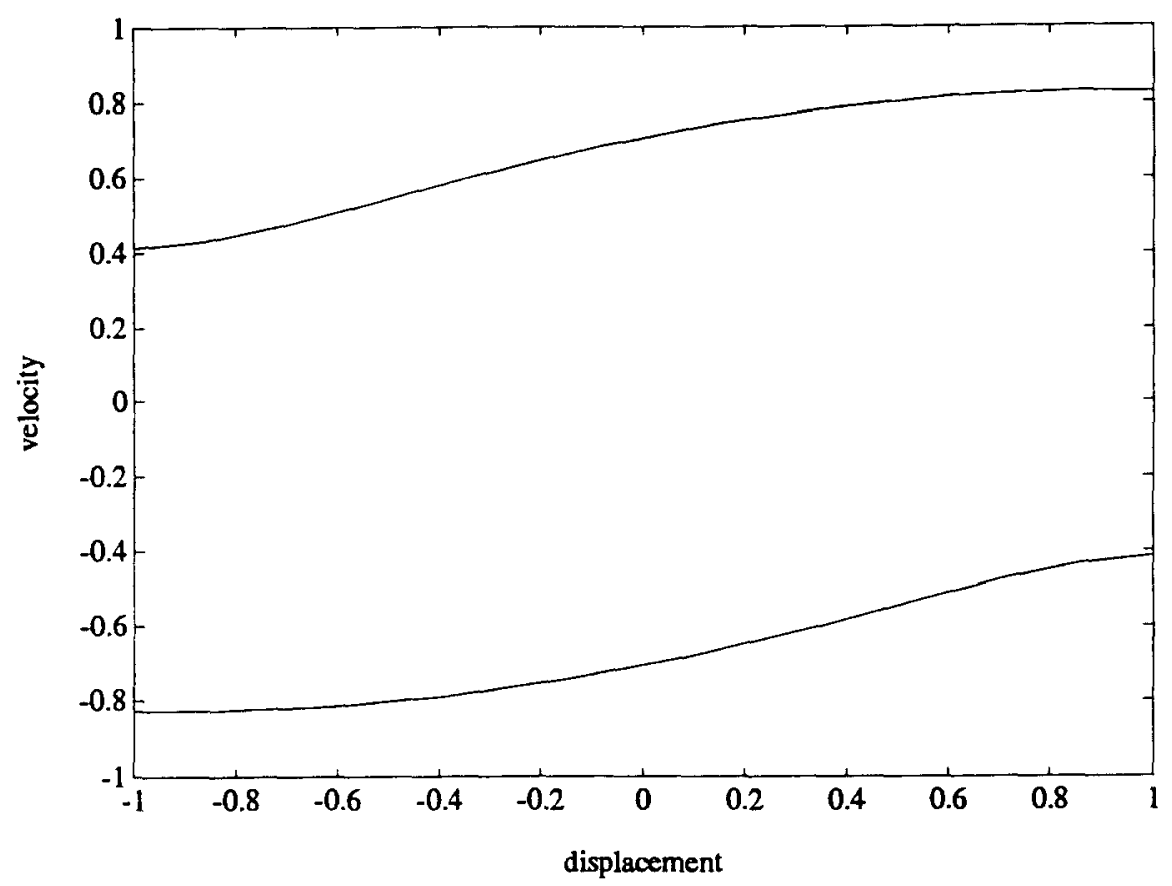

Fig. 2. Periodic motion in the $x, x^{\prime}$ plane for $r=0.5, \gamma=0.20826$, obtained by Li et al. [5].

\section{Simple Cell Mapping}

\subsection{Method Explanation}

Under the SCM method, a particular region $\Omega$ in the state space, called the region of interest, is discretized into a finite number of cells, say $M$, with index $1, \ldots, M$. The remaining part of the state space is called the sink cell and has index 0 . Each cell represents an indivisible state entity. The state of the system is described by a cell index $\xi \in\{0, \ldots, M\}$ instead of a state vector $\mathrm{x}=\left(x_{1}, \ldots, x_{N}\right)$, where $N$ is the state space dimension.

The evolution of a system can be described as a sequence of cells, by inspecting its state at discrete equidistant times. Let $\xi(n)$ denote the cell containing the state of the system at $t=n T, n=0,1, \ldots$, with $T$ the time between two state inspections. The system evolution is then governed by

$$
\xi(n+1)=C(\xi(n)),
$$

where $C: \mathrm{N} \rightarrow \mathrm{N}$ is called a Simple Cell Mapping. For periodic systems, $T$ should be chosen equal to the system's period to obtain a SCM $C$ which is independent of $n$.

We distinguish between cells which are periodic, i.e., cells $\xi^{*}$ with $C^{m}\left(\xi^{*}\right)=\xi^{*}$, for some $m \in \mathbb{N}$ which is called the period of $\xi^{*}$, and cells which are not. These cells are called transient cells, and have the chance of being mapped onto a periodic cell, in a finite number of steps, or onto the sink cell. By definition, the system will then stay there forever.

Groups of periodic cells represent the system's recurrent states (attractors, saddles and repellors). Although no aperiodic motion can occur because of the finite number of cells, chaotic motion can be expected when dealing with periodic groups of relatively long period. For more details about the SCM method, see Hsu [2]. 


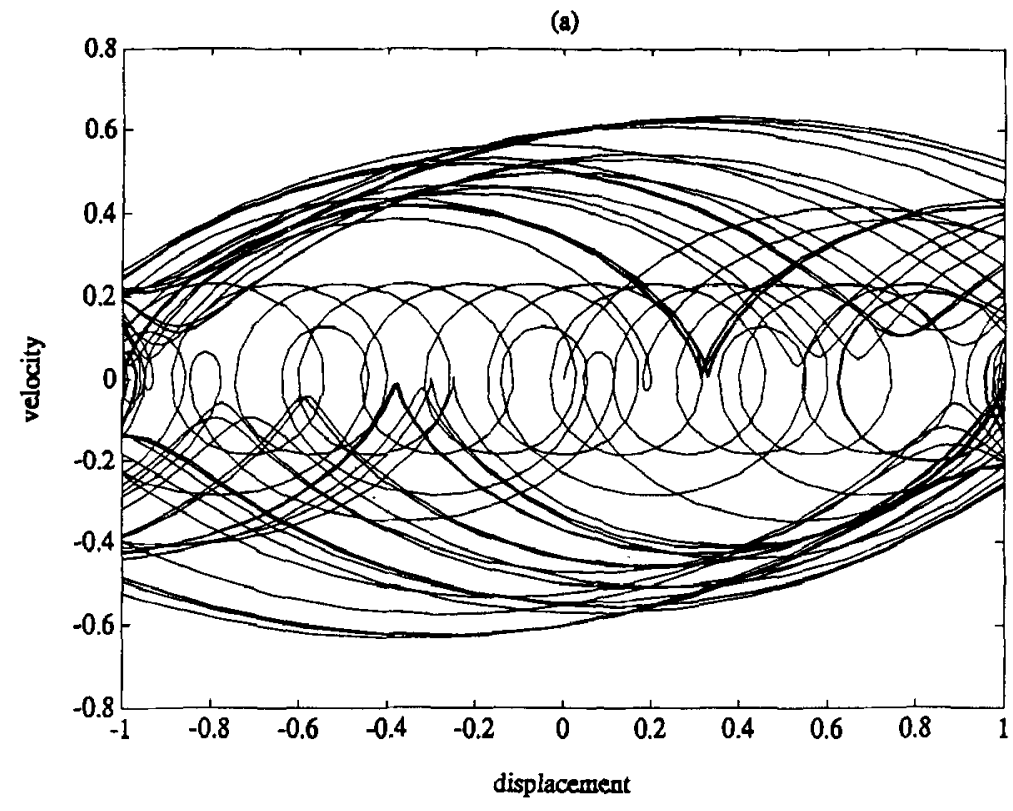

(b)

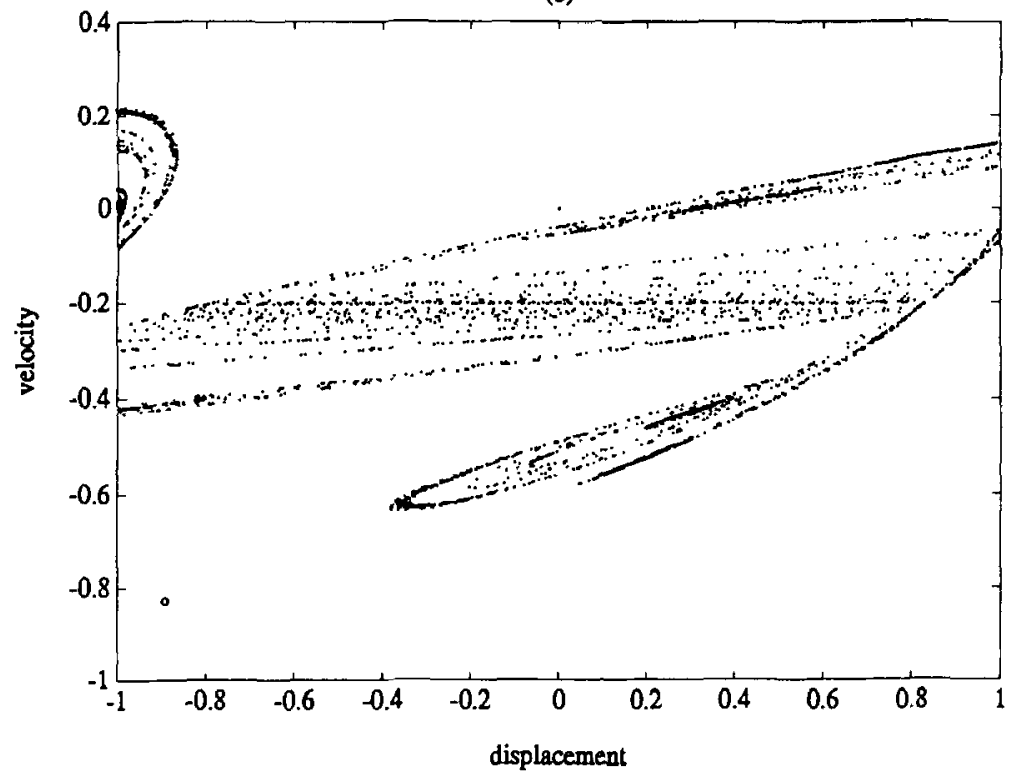

Fig. 3. (a) Chaotic motion in the $x, x^{\prime}$ plane for $r=0.5, \gamma=0.20826$. (b) Poincare section of periodic (o) and chaotic $(\cdot)$ motions.

\subsection{APPLICATION: IMPACT OSCILLATOR}

To apply the SCM method to the impact oscillator, we first have to choose our region of interest $\Omega$. Obviously, for $x$ we have $|x| \leq 1$. For convenience, we also choose $\left|x^{\prime}\right| \leq 1$. Next, we discretize $\Omega$ by dividing it into $101 \times 101(=10201)$ rectangular cells of equal magnitude, with index $1,2, \ldots, 10201$. The determination of every cell's image cell is done by the center point method: we take the center point of each cell and execute a numerical integration of (1) 
over one forcing period $T=2 \pi$ with this point as initial condition. The image cell is then given by the cell which contains the end point of the integrated trajectory.

When integrating (1), we have to be aware of the discontinuity in the velocity at $|x|=1$. We must integrate until $|x|=1$, change the velocity $x^{\prime}$ into $-r x^{\prime}$, and continue integrating until $|x|=1$ again. To realize this procedure, we use the Hénon method (see [1]). Here, the Hénon method means rearranging (1) in such a way that $x$ becomes the independent variable and $t$ the dependent one. We achieve this by writing the equation of motion in (1) as a first order system:

$$
\begin{aligned}
\frac{\mathrm{d} x_{1}}{\mathrm{~d} t} & =x_{2}, \\
\frac{\mathrm{d} x_{2}}{\mathrm{~d} t} & =\gamma \sin (t) .
\end{aligned}
$$

Next, we divide the second equation by the first one and invert the first one:

$$
\begin{aligned}
& \frac{\mathrm{d} t}{\mathrm{~d} x_{1}}=\frac{1}{x_{2}} \\
& \frac{\mathrm{d} x_{2}}{\mathrm{~d} x_{1}}=\frac{1}{x_{2}} \gamma \sin (t) .
\end{aligned}
$$

To describe the procedure followed during numerical integration, we introduce the following definitions:

$t^{j} \quad:$ value of $t$ after $j$-th integration step

$\mathrm{x}^{j} \quad$ : calculated value of $\mathbf{x}=\left(x_{1}, x_{2}\right)$ after $j$-th integration step

$x_{i}^{j} \quad$ : calculated value of $x_{i}(i=1,2)$ after $j$-th integration step

$h^{j} \quad$ : applied stepsize in $j$-th integration step

We start the numerical integration with system (3). When for some $j$ we have $\left|x_{1}^{j}\right|>1$, we switch to system (4) and carry out one integration step with initial condition $\left(t^{j-1}, x_{2}^{j-1}\right)$ and stepsize $x_{1}=\operatorname{sgn}\left(x_{1}^{j}\right)-x_{1}^{j-1}$. In this way, we easily obtain the values of $t$ and $x_{2}$ at $\left|x_{1}\right|=1$. After multiplying $x_{2}$ by $-r$ we continue integrating system (3) until we have $\left|x_{1}\right|>1$ again. However, when $x_{2}$ is small, (4) becomes a set of stiff differential equations, which are hard to integrate. For $x_{2}=0$ the system derivatives are even infinitely large. Hence, the Hénon method cannot be applied for small values of $x_{2}$.

When $x_{2}$ is too small, we use a less sophisticated but more robust way to integrate over the discontinuity at $\left|x_{1}\right|=1$. When for some $j$ we have $\left|x_{1}^{j}\right|>1$ and $x_{2}^{j-1}$ is small, we perform a new integration from $\mathrm{x}^{j-1}=\left(x_{1}^{j-1}, x_{2}^{j-1}\right)$ with stepsize $h_{1}^{j}=\frac{1}{2} h^{j}=\frac{1}{2}\left(t_{j}-t_{j-1}\right)$ which yields a new $x_{1}^{j}$. Next, a new integration is carried out with stepsize $h_{2}^{j}$, with

$$
h_{2}^{j}=\left\{\begin{array}{l}
h_{1}^{j}+h^{j} / 4 \text { if }\left|x_{1}^{j}\right|<1, \\
h_{1}^{j}-h^{j} / 4 \text { if }\left|x_{1}^{j}\right|>1 .
\end{array}\right.
$$

This procedure is repeated until ||$x_{1}^{j}|-1|<10^{-3}$. Since every integration starts from the same state, no accumulation of integration errors will appear.

This stepsize-halving method was used when $x_{2}<0.05$; the Hénon method in all other cases. Numerical integration was done with a fourth-order Runge-Kutta scheme. Used parameters were: initial stepsize $h_{\text {start }}=10^{-2}$, minimum stepsize $h_{\min }=10^{-4}$, and maximum local 


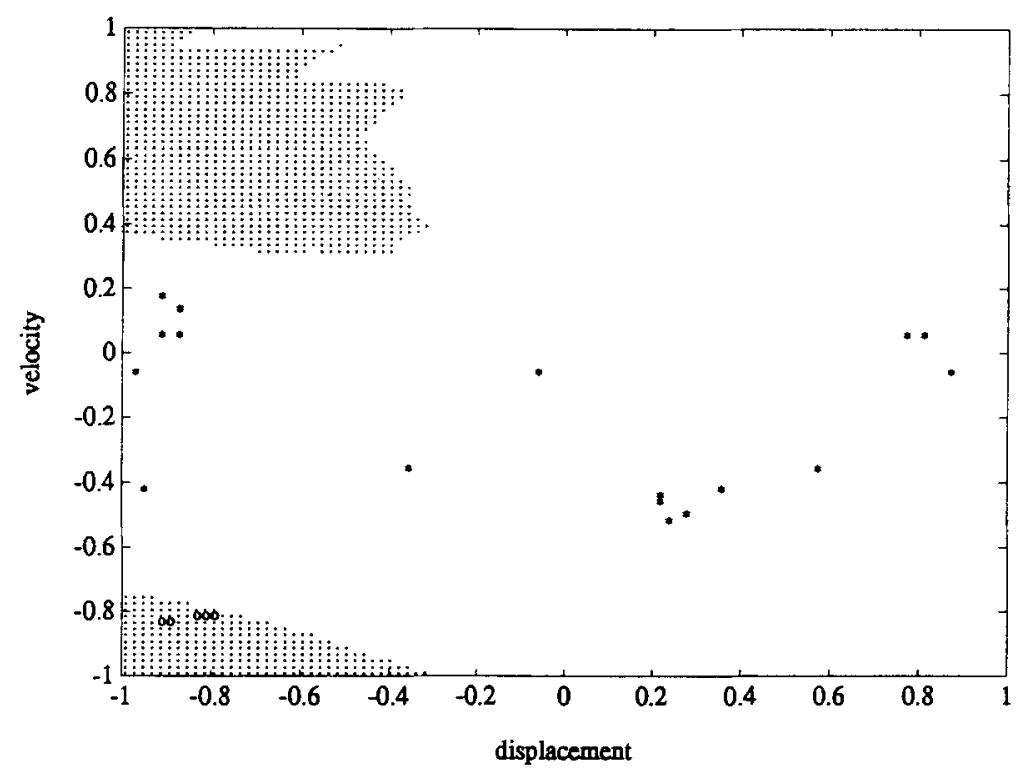

Fig. 4. Attractors and basins of attraction obtained by SCM: $P-1$ cells $(0)$ and their transient cells $(\cdot) ; P-17$ group $(*)$ and its transient cells (white).

truncation error $\epsilon=10^{-4}$. A smaller value of $\epsilon$ does not give us better results; we are only interested in the cell which contains a trajectory's end point, not in the exact position of this point itself.

\subsection{RESULTS}

Figure 4 shows the results of the SCM method applied to the impact oscillator, using the above mentioned technique to overcome discontinuities during numerical integration. The periodic solution is represented by 5 clustered cells of period $1(0)$. The transient cells $(\cdot)$ leading to one of these cells together form the basin of attraction of the periodic solution. The chaotic solution is represented by 17 cells of period $17(*)$, forming one group. This group appears to be an attractor because of its huge number of transient cells (the white area in Figure 4).

To check the correctness of these results, we carried out a complete numerical integration for all cell center points. Here, "complete" means that integration was continued until convergence was reached (convergence criterion: $\epsilon=10^{-6}$ ), with a maximum integration time of $40 T$ $(80 \pi)$ seconds. If no convergence was obtained within this time limit, the trajectory was considered to be chaotic.

Figure 5 shows the "exact" results: A periodic solution (o) at $(-0.893717,-827896)$, its basin of attraction $(\cdot)$, and the chaotic attractor, which is produced by the end points of all chaotic trajectories. With the exception of the chaotic attractor, the SCM results reasonably match with the exact results. 


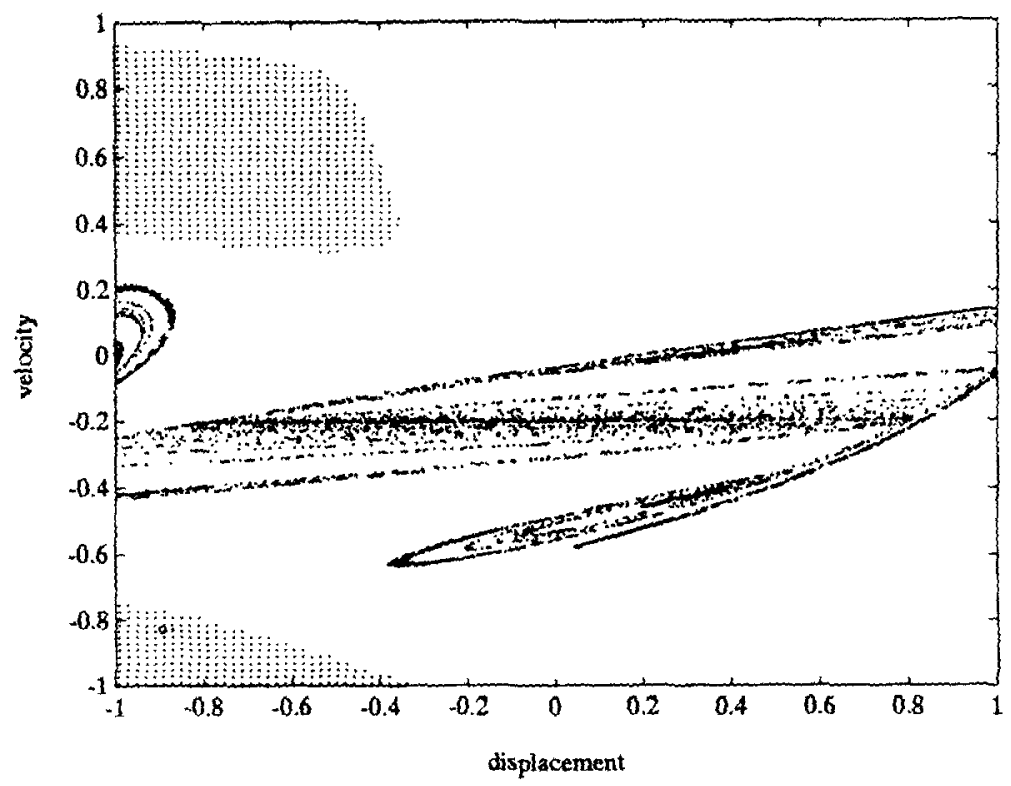

Fig. 5. Attractors and basins of attraction obtained by numerical integration: Periodic attractor (o) and its basin of attraction (.); chaotic attractor $(\cdot)$ and its basin of attraction (white).

\section{Generalized Cell Mapping}

\subsection{Method Explanation}

Under the GCM method, as opposed to SCM, a cell is allowed to have more than one image cell. Each cell is mapped onto one of its image cells with a fraction of the total probability. If $M$ is the number of cells, the state of the system at $t=n T$ is described by the cell probability vector $\mathbf{p}(n)=\left[p_{1}(n) . . p_{M}(n)\right]^{T}$, with

$$
p_{i}(n)=\operatorname{Prob}\{\text { system is in cell } i \text { at } t=n T\}, \quad n \in \mathbb{N} .
$$

The system evolution is then given by the equation

$$
\mathrm{p}(n+1)=\mathrm{P} \mathrm{p}(n)
$$

where $\mathbf{P}=\left(p_{i j}\right)$ is the transition probability matrix:

$$
p_{i j}=\operatorname{Prob}[\text { system is in cell } i \text { at } t=(n+1) T / \text { system is in cell } j \text { at } t=n T] \text {. }
$$

Equation (7) describes a finite, discrete, stationary Markov chain (see e.g., Isaacson and Madsen [4]). According to the theory of Markov chains, we can divide the cells into persistent and transient cells. A persistent cell $i$ has the property that when the system is in $i$ at a certain moment, it will return to $i$ at some time in the future. All persistent cells can be formed into several persistent groups ( $\mathrm{PG}$ 's), which are closed. This means that when the system enters a particular PG, it will stay in there forever and cannot enter any other PG. The PG's of a Markov chain, deduced from a dynamical system, correspond to the attractors of that system.

When a cell is not persistent, it is transient by definition. For finite Markov chains, the system will leave the transient cells with probability one and will settle on one of the PG's. The transient cels can be divided into single- and multiple-domicile cells. Starting in a singledomicile cell, the system will lead to a particular PG with probability one; hence these cells 


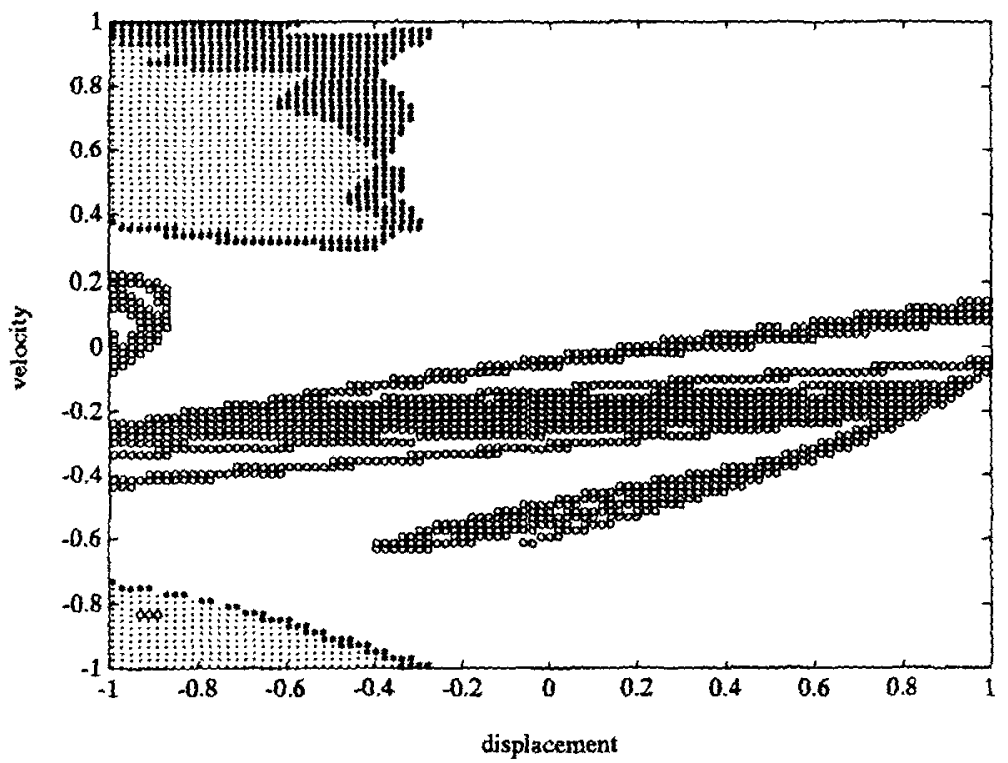

Fig. 6. Results obtained by GCM: Persistent groups (o), single domicile cells leading to the periodic $(\cdot)$ and the chaotic (white) attractor, and multiple domicile cells $\left({ }^{*}\right)$.

form the basins of attraction of the PG's. Starting in a multiple-domicile cell, the system can lead to several PG's with corresponding probabilities, the sum of which of course equals one. The multiple domicile cells form the separatrices, the boundaries between the basins of attraction.

\subsection{APPLICATION: IMPACT OSCILLATOR}

To apply the GCM method to the impact oscillator, we take the same region of interest $\Omega$ as under SCM and again divide it into 10201 cells. To approximate the transition probabilities we use the sampling method: For each cell $j, 9$ uniformly distributed sampling points are chosen, including the center point. For each sampling point, a numerical integration is carried out over one forcing period $T=2 \pi$. The transition probability $p_{i j}$ corresponding to a certain image cell $i$ is simply determined by the fraction of end points lying in $i$. For the numerical integration, we again use the technique described in the previous section.

\subsection{RESULTS}

Figures 6 and 7 show the results of the GCM method applied to the impact oscillator. Two persistent groups are obtained: one consisting of 3 cells, corresponding to the periodic solution, and one consisting of 1324 cells, corresponding to the chaotic solution. In Figure 6 these groups have been plotted $(0)$, together with the multiple domicile cells $(*)$ and the basin of attraction of the periodic solution $(\cdot)$. The basin of attraction of the chaotic solution consists of all other cells (left blank in Figure 6 ).

Further, for each cell of each $P G$ the limiting probability was determined, which is the probability of the system being in that cell at $t \approx \infty$, under the condition that the system entered the corresponding PG. For the chaotic solution, we expressed this probability in the mean value MV which equals 1/1324 (Figure 7). Cells denoted by o have a limiting probability 


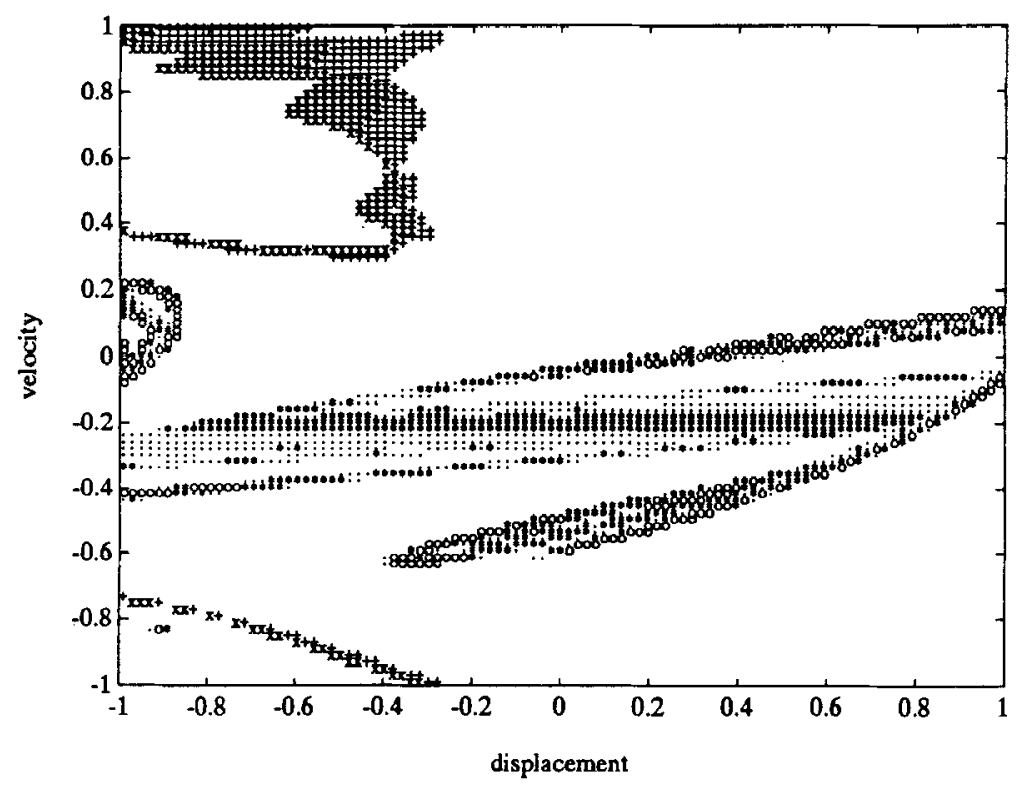

Fig. 7. Probabilistic properties of persistent groups and muitiple domicile cells, obtained by GCM: MD cells leading to the periodic $(x)$ and the chaotic $(+)$ attractor with probability $p>0.5$. Persistent cells having a limiting probability $p=f * \mathrm{MV}$ (Mean Value), with $f>1.5(0), 0.5 \leq f \leq 1.5(*)$, and $f<0.5(\cdot)$.

of $f * M V$, with $f>1.5$. For cells denoted by $*$ and ', we have $f \in[0.5,1.5]$ and $f \in[0.0,0.5]$ respectively. The three cells corresponding to the periodic solution, denoted by $\circ, *$, and ?, have a limiting probability of $0.52,0.39$, and 0.09 respectively.

Finally, to obtain a better picture of the basins of attraction, the multiple domicile cells were divided in two groups: cells leading to the periodic solution with probability $p>0.5$ $(\times)$ and cells leading to the chaotic solution with probability $p>0.5(+)$ (Figure 7).

When we compare these results with the "exact" ones (Figure 5), we see that the basins of attraction are found to be slightly more accurate than with SCM (when we add the MD cells that lead to a particular attractor with probability $p>0.5$ ). The large persistent group gives a good picture of the chaotic attractor, especially when the limiting probability distribution of this group is taken into account.

\section{Interpolated Cell Mapping}

\subsection{METHOD EXPLANATION}

The ICM method was introduced by Tongue [6] as an improvement on SCM, taking only slightly more CPU-time. Just as in SCM, a numerical integration is carried out over one forcing point for a huge number of initial states. These states should no longer be seen as cell center points but as interpolation points. The system evolution is no longer described by a sequence of cells but by ordinary state coordinates. When the state of the system at $t=n T$ is given by $\mathbf{x}_{n}=\left(x_{n}, x_{n}^{\prime}\right)$, the state at $t=(n+1) T$ is obtained by a bilinear interpolation of $\mathbf{x}_{n}$ between the image points of the four gridpoints surrounding $\mathbf{x}_{n}$. For an arbitrary initial state, a complete trajectory can be constructed in this way. For a more detailed explanation of the ICM method, we refer to Tongue [6]. 


\subsection{APPLICATION: IMPACT OSCILLATOR}

To apply the ICM method to the impact oscillator, we need a grid of points distributed over $\Omega$. Since every state in $\Omega$ must have four surrounding grid points, the grid points must lie on the boundary of $\Omega$ as well. Therefore, we cannot use the center points of the cells used under SCM, since these do not lie on the boundary of $\Omega$. Hence, we have to perform an additional numerical integration for a completely new grid, given by $\left(i h_{1}, j h_{2}\right), i, j=-50, . ., 50, h_{1}=h_{2}=0.02$. Further, we have to take a smaller value for the maximum local truncation error $\epsilon$, since now we are interested in the exact position of the image point of each grid point. Here, we take $\epsilon=10^{-6}$.

Because of the discontinuity in (1), we deal with an additional difficulty. Since the interpolation only gives a good approximation of reality when the end points of the four trajectories are not too far apart, it can only be applied when all four trajectories undergo an equal number of impacts. When this is not the case, an ordinary numerical integration should be carried out instead of the bilinear interpolation. Of course, all integrations have to be executed with the modified integration procedure, given in Section 3, to overcome the discontinuities.

\subsection{RESULTS}

Figure 8 shows the results of applying the modified ICM method to the impact oscillator. We find the periodic solution at $(-0.894982,-0.828017)(0)$ and its basin of attraction $(\cdot)$. Further, we find a periodic solution at $(-0.762251,-0.806073)(+)$, which appears to be a saddle solution. We can see this from its basin of attractin $(x)$, which is formed by 39 initial states, all lying on the separatrix. This separatrix separates the basins of attraction of the two attractors. Trajectories that do not show convergence $\left(\epsilon=10^{-3}\right)$ within 40 interpolation/integration steps are assumed to end up on the chaotic attractor (see Figure 8). Its basin of attraction is of course given by the initial states of these trajectories (the white area in Figure 8).

When we compare these results with the "exact" ones, we see that the periodic solution is found almost exactly, just as is its basin of attraction. The chaotic attractor also matches with the one obtained by numerical integration very well. Additionally, the determination of the saddle solution is an important result, specially when one is interested in bifurcation research, in which saddle solutions play an important role.

\section{Mixed Cell Mapping}

\subsection{METHOD EXPLANATION}

One of the variations on ICM is given by the Multiple Mapping (MM) method, introduced by Tongue [8]. Under MM, the regular map over one forcing period is replaced by several maps, each covering a small time interval. For instance, under Bi-Mapping two maps are applied to obtain an image point. The first map covers $0 \leq t \leq T / 2$. The second map covers $T / 2 \leq t \leq T$.

The idea behind this variation on ICM is the expectation that a shorter interpolation interval will improve the accuracy of the trajectory determination (Tongue [8]). However, this does not need to be true. In fact, halving the interpolation interval makes only sense for states that are being interpolated between trajectories that have diverged in some way at $t=T / 2$. 


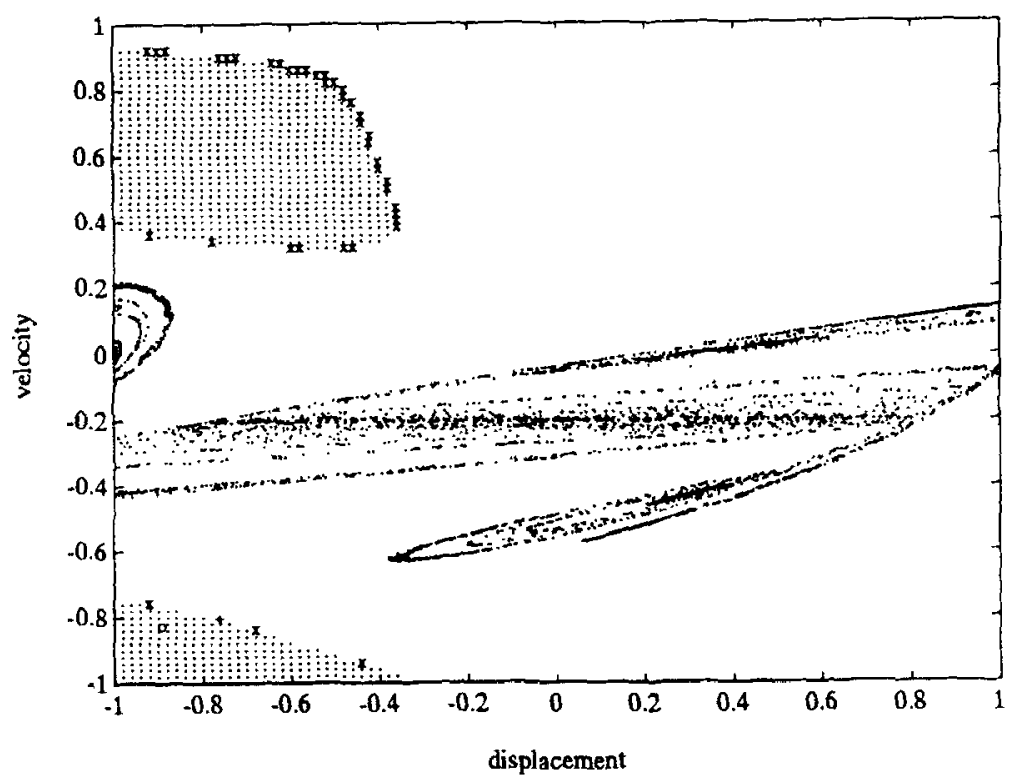

Fig. 8. Results obtained by ICM: Stable periodic solution (o) and its basin of attraction (.); saddle solution ( + ) and its stable manifold $(x)$; chaotic attractor $(\cdot)$.

Therefore, we introduce a combination of MM and ICM, termed Mixed Cell Mapping (MCM). Under MCM, a regular ICM is used unless we deal with one of the following situations: (a) The interpolation trajectories have diverged at $t=T / 2$ with respect to the original grid; (b) The interpolation trajectories enclose a concave area at $t=T$. In both cases we use MM (Bi-Mapping) to obtain the next image point.

\subsection{APPLICATION: IMPACT OSCILLATOR}

We applied the MCM method to the impact oscillator, using the same grid and criteria as under ICM. Further, divergence of interpolation trajectories was defined to take place when $d>1$, with

$$
d=\frac{1}{3}\left(\frac{d_{1}}{h_{1}}+\frac{d_{2}}{h_{2}}+\frac{d_{3}}{h_{3}}\right)
$$

Here, $h_{i}$ and $d_{i}(i=1,2,3)$ represent the distances between four interpolation trajectories at $t=0$ and $t=T / 2$ respectively, as is shown in Figure 9. For an $N$-dimensional system $(N>2)$, the divergence can be generalized by including the distances between the trajectory with the smallest coordinates in the reference situation and the $2^{N}-1$ other interpolation trajectories at $t=0$ and $t=T / 2$.

Since for each grid point we had to perform a numerical integration for $t=0$ to $t=T$ and one for $t=T / 2$ to $t=T$, the amount of CPU-time for the integration part was enlarged with a factor 1.5 with respect to ICM. However, due to the use of two maps instead of one (in many cases), the situation of four trajectories crossing the discontinuity an unequal number of times was encountered only half as often as under ICM. So, we obtained a (slightly) more accurate result than under ICM for even a smaller (CPU-)price. 


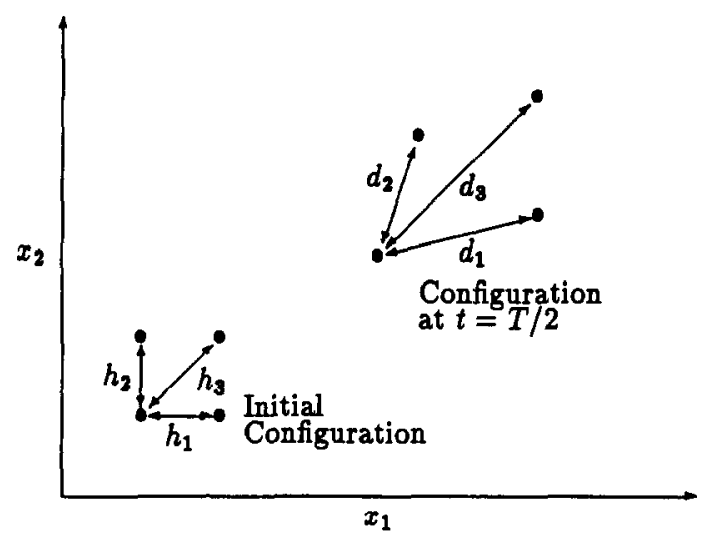

Fig. 9. Definition of state space divergence.

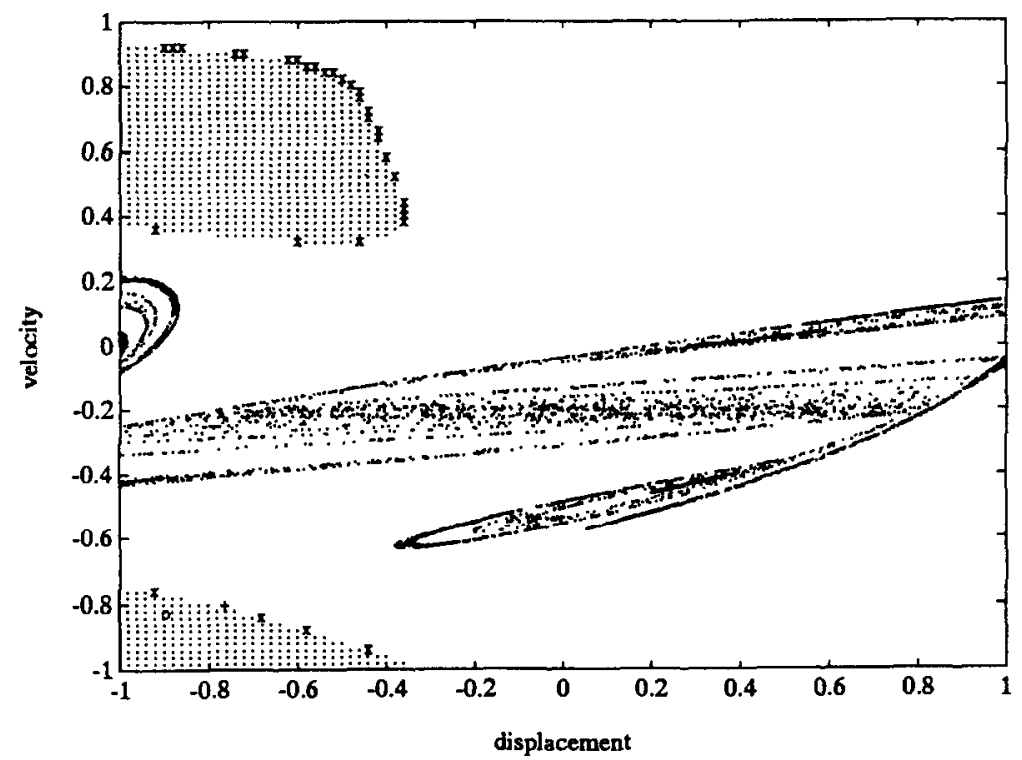

Fig. 10. Results obtained by MCM: Stable periodic solution (o) and its basin of attraction ( $\cdot)$; saddle solution (+) and its stable manifold $(x)$; chaotic attractor $(\cdot)$.

\subsection{RESULTS}

Figure 10 shows the results of the MCM method applied to the impact oscillator. We find the periodic solution at $(-0.895816,-0.828167)(0)$ and its basin of attraction $(\cdot)$. Just as under ICM, we find the saddle solution, at $(-0.762908,-0.806236)(+)$, and its basin of attraction $(x)$. In the same way as under ICM, the chaotic attractor and its basin of attraction are determined (see Figure 10).

When we compare these results with those obtained by ICM, we hardly see any difference. This is because under ICM use has been made of integration instead of interpolation many times, which improves of course the accuracy of the results. But, compared to ICM, an obvious gain in CPU-time has been achieved. 
Table I. Computational aspects

\begin{tabular}{lrc}
\hline Method & CPU(s) & Storage $(\mathrm{Kb})$ \\
\cline { 2 - 3 } SCM & 27 & 252 \\
GCM & 277 & 9404 \\
ICM & 572 & 600 \\
MCM & 524 & 1552 \\
EXACT & 2061 & 400 \\
\hline
\end{tabular}

To conclude this comparison of cell mapping methods, the required CPU-times of the applied methods are given in Table I, together with the storage requirements. The simulations were done on a Silicon Graphics 4D30 workstation.

\section{Conclusion}

A modification has been added to the existing cell mapping techniques, termed Mixed Cell Mapping (MCM). MCM is a combination of ICM and MM (Multiple Mapping), producing more accurate results at the cost of more CPU-time. When applying MCM to discontinuous systems, even a gain in CPU-time may be achieved.

It has been shown that cell mapping techniques can be applied to discontinuous dynamic systems. For that purpose, some ad-hoc modifications need to be made in the numerical integration part. To apply the ICM and MCM methods, one needs to replace the interpolation by integration when the trajectories starting from the interpolation points do not cross the discontinuity an equal number of times.

For the special case of the impact oscillator, the MCM and ICM methods produced the best results. However, because of the extra integration steps, even more CPU-time was required than under GCM. Application of the GCM method is recommended when one is particularly interested in probabilistic properties of the system one is investigating.

\section{References}

1. Hénon, M., 'On the numerical computation of Poincaré maps', Physica D 5, 1982, 412 414.

2. Hsu, C. S., 'A theory of cell-to-cell mapping dynamical systems', Joumal of Applied Mechanics 47, 1980, 931-939.

3. Hsu, C. S., Cell-to-Cell Mapping; A Method of Global Analysis for Nonlinear Systems, Springer-Verlag, New York, Heidelberg, Berlin, Tokyo, 1987.

4. Isaacson, D. L. and Madsen, R. W., Markov Chains: Theory and Applications, John Wiley \& Sons, New York, London, Sidney, Toronto, 1976.

5. Li, G. X., Rand, R. H., and Moon, F. C., 'Bifurcation and chaos in a forced zero-stiffness impact oscillator', International Journal of Non-Linear Mechanics 25, 1990, 417-432.

6. Tongue, B. H., 'On obtaining global nonlinear system characteristics through interpolated cell mapping', Physica D 28, 1987, 401-408.

7. Tongue, B. H., 'Interpolated cell mapping of dynamical systems', Journal of Applied Mechanics 55, 1988 , 461-466.

8. Tongue, B. H., 'A multiple-map strategy for interpolated mapping', International Journal of Non-Linear Mechanics 25, 1990, 177-186. 